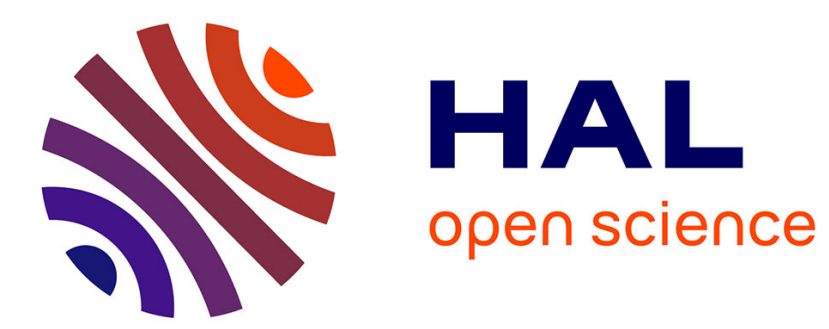

\title{
Simulating a diffusion on a graph. Application to reservoir engineering
}

Antoine Lejay

\section{To cite this version:}

Antoine Lejay. Simulating a diffusion on a graph. Application to reservoir engineering. Monte Carlo Methods and Applications, 2003, 9 (3), pp.241-255. inria-00092428

\section{HAL Id: inria-00092428 \\ https://hal.inria.fr/inria-00092428}

Submitted on 10 Sep 2006

HAL is a multi-disciplinary open access archive for the deposit and dissemination of scientific research documents, whether they are published or not. The documents may come from teaching and research institutions in France or abroad, or from public or private research centers.
L'archive ouverte pluridisciplinaire HAL, est destinée au dépôt et à la diffusion de documents scientifiques de niveau recherche, publiés ou non, émanant des établissements d'enseignement et de recherche français ou étrangers, des laboratoires publics ou privés. 


\section{Simulating a diffusion on a graph. Application to reservoir engineering}

Antoine Lejay ${ }^{1, \dagger}$ — Projet OMEGA (INRIA / Institut Élie Cartan, Nancy)

Abstract: We develop a simple Monte Carlo method to compute the position at a given time of a diffusion on a graph, with constant speed on each edge. This method is exact, and we claim it could be used for simulating the position of a particle in a fissured media. Besides, we advocate that the notion of diffusions on graphs could be useful to understand the behavior of onedimensional diffusions whose infinitesimal generator has piecewise constant coefficients.

Keywords: diffusion on graphs, skew brownian motion, onedimensional diffusion processes, Monte Carlo methods

AMS Classification: 65C, 60K40

Published in Monte Carlo Methods Appl.. 9:3, 241-256, 2003

${ }^{1}$ Current address: IECN

Campus scientifique

B.P. 239

54506 Vandœuvre-lès-Nancy CEDEX (France)

E-mail: Antoine.Lejay@iecn.u-nancy.fr

${ }^{\dagger}$ This work has been partially supported by the "Groupe de Recherche MOMAS" financed by the french institutions ANDRA, BRGM, CEA, CNRS and EDF. 
A. Lejay / Simulating a diffusion on a graph. Application to reservoir engineering

\section{Introduction}

A diffusion on a graph could be seen as an extension of a one-dimensional stochastic process, whose behavior at some points (the vertices) is specified. Such stochastic processes were introduced first by G. Lumer in [LUM84].

Diffusions on graphs arise naturally both in physical models and in mathematical methods. For example, they could be obtained as limit of some averaging, as in [FW93]. Besides, diffusions on graphs have attracted more interest in the mathematical community during the last decade due to the works of M. Freidlin and M. Weber on perturbed Hamiltonian systems (See for example [FW98, FS00],...).

In this article, we give an exact algorithm to simulate diffusions on graphs for which the diffusion coefficient is constant on each edge. Our algorithm follows in fact a description of the dynamic of the diffusion process.

Our motivation comes from reservoir engineering. A fluid in movement in a fissured porous media tend to move essentially in the fissures, since the permeability is much more greater there. Thus, the question is to know how a fluid reaches a given surface is dramatically conditioned by the geometry of the fissures (See for example [DJ93] for an example where choice of the parameters defining the geometry of a comb leads to different results).

The algorithm is the sequel of a work concerning Monte Carlo methods for simulating the displacement of a fluid in a fissured porous media without using approximation by random walks: See CL01, CL02.

In Section 5, we invoke an averaging result by M. Freidlin and A.D. Wentzell [FW93] to show that if the porous media is assumed to have a permeability equal to 0 , then our algorithm could be used as a Monte Carlo method for simulating the movement of a fluid particle in a two dimensional fissures' network. In this approach, we assume that the fissures have smooth boundaries. This does not correspond to the reality, where the fissures are rather assumed to have a fractal boundary. But our model could be obtained by considering effective coefficients.

In a forthcoming work, we deal with the case of a porous media with a low permeability, and our approach could be used to characterize the exchange of particles between the fissures and the porous media. Other extensions could be considered, in order to deal with more realistic models. Adding a drift term can also be considered.

Before giving a description of our algorithm, we gather in Section 2 some results about diffusions on graphs. Besides, we claim that the concept of diffusions on graph could help to understand the behavior of one-dimensional processes whose infinitesimal generators (in divergence form or not) has coefficients that are discontinuous at a few points and smooth elsewhere. Thus, 
A. Lejay / Simulating a diffusion on a graph. Application to reservoir engineering

we continue some of the work presented in LEJ00, LEJ01 concerning the understanding of the behavior of a process when it reaches some points at which the diffusion coefficient of its infinitesimal generator is discontinuous.

\section{Diffusions on a graph}

\subsection{Definition of a graph and notations}

Let $G$ be an directed graph. Each edge $e$ could be seen as an arrow that connects a vertex $v_{e}$ to a vertex $w_{e}$.

Hypothesis 1. To each edge, we associate a positive real number $\ell_{e}$ called the length of $e$. Possibly, $\ell_{e}$ may be infinite, but in this case, we assume that there exists a vertex $v^{\infty}$ for which $\ell_{e}=+\infty$ is equivalent to $w_{e}=v^{\infty}$. This vertex plays the role of the "point at infinity". We also assume that

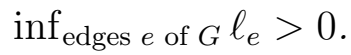

Once the orientation and the length of an edge $e$ are given, $e$ could be seen as the interval $I_{e}=\left[0, \ell_{e}\right]$. We identify 0 with $v_{e}$ and $\ell_{e}$ with $w_{e}$. For two points $x$ et $y$ of $e$, we use the usual Euclidean distance $d(x, y)=|x-y|$.

An edge $e$ is said to be incident to a vertex $v$ if either $v_{e}=v$ or $w_{e}=v$. We also use the notation $e \sim v$ when $e$ is incident to $v$.

Hypothesis 2. For simplicity, we assume that the graph $G$ is connected. We assume that the degree of $v$, that is the number of edges that are incident to $e$, is finite for each vertex, although there could be an infinite number of edges.

\subsection{Diffusion on a graph}

A diffusion on a graph is a continuous, conservative, strong Markov stochastic process. This process will be defined by its infinitesimal generator.

Let us denote by $\mathfrak{I}$ the space $\cup_{\text {edge } e}\left(e, I_{e}\right)$. By a point $x$ in $\mathfrak{I}$, we mean a couple made of an edge $e$ and of a point in $\mathbb{R}_{+}$. Let us denote by $\mathcal{C}(\mathfrak{I})$ the set of continuous, real functions on $\mathfrak{I}$, which means that $f(e, \cdot)$ is continuous on each $\left(0, \ell_{e}\right)$ (using the canonical identification of $e$ with $I_{e}$ ) and for each vertex $v$, there exists a real $c_{f, v}$ such that $c_{f, v}=\lim _{x \rightarrow 0} f(e, x)$ if $v=v_{e}$ and $c_{f, v}=\lim _{x \rightarrow \ell_{e}} f(e, x)$ if $v=w_{e}$. When there is no ambiguity, we write $f(x)$ instead of $f(e, x)$ for functions on $\mathfrak{I}$.

For each vertex $v$ and each edge $e$ incident to $v$, we denote by $\mathcal{D}_{v, e}$ the following derivation operator: for $x$ in $\left(0, \ell_{e}\right)$,

$$
\mathcal{D}_{v, e} f(x)=f^{\prime}(x) \text { if } v=v_{e} \text { and } \mathcal{D}_{v, e} f(x)=-f^{\prime}(x) \text { if } v=w_{e},
$$


and $\mathcal{D}_{v_{e}, e} f\left(v_{e}\right)=f^{\prime}(0+), \mathcal{D}_{v_{e}, e} f\left(w_{e}\right)=f^{\prime}\left(\ell_{e}-\right), \mathcal{D}_{w_{e}, e} f\left(w_{e}\right)=-f^{\prime}\left(\ell_{e}-\right)$ and $\mathcal{D}_{w_{e}, e} f\left(v_{e}\right)=-f^{\prime}(0+)$. Here, where $f^{\prime}(x)$ is the usual derivative of $f(e, x)$ of $f$ at the point $x$ in $\left(0, \ell_{e}\right)$ and $f^{\prime}(x+)=\lim _{y \rightarrow x, y<x} f^{\prime}(y)$ and $f^{\prime}(x-)=$ $\lim _{y \rightarrow x, y>x} f^{\prime}(y)$. This operator could be easily applied many times, and we denote by $\mathcal{C}^{k}(\mathfrak{I})$ the space of continuous, real functions, with continuous derivatives up to order $k$ on each $\left(0, \ell_{e}\right)$. Note that with this definition, the derivatives of $f$ are not required to be continuous at the vertices.

At each edge $e$, we associate two measurable functions $a_{e}$ and $b_{e}$ from $I_{e}$ to $\mathbb{R}$ such that $b_{e}$ is bounded and $c<\inf _{x \in I_{e}} a_{e}(x) \leq \sup _{x \in I_{e}} a_{e}(x)<C$, where $0<c<C$, and the previous inequalities are uniform with respect to the edges.

We consider the differential operator

$$
L f(x)=\frac{1}{2} a_{e}(x) \mathcal{D}_{v_{e}, e}^{2} f(e, x)+b_{e}(x) \mathcal{D}_{v_{e}, e} f(e, x)
$$

defined for bounded functions $f$ in $\mathcal{C}^{2}(\mathfrak{I})$. To specify fully the generator, we also need to express the domain of the operator $L$.

We associate to each vertex $v$ and each edge $e$ incident to $v$ some reals numbers $\alpha_{v, e}$ such that

$$
0 \leq \alpha_{v, e} \leq 1 \text { and } \sum_{e \sim v} \alpha_{v, e}=1
$$

The real number $\alpha_{v, e}$ are called weights.

Thus, the domain $\operatorname{Dom}(L)$ of $L$ is the set of bounded functions $f$ in $\mathcal{C}^{2}(\mathfrak{I})$ such that

$$
\sum_{e \sim v} \alpha_{v, e} \mathcal{D}_{v, e} f(v)=0 .
$$

This condition (3) is called the pasting condition.

The next theorem is proved using the martingale problem. See [FW93] for example.

Theorem 1. If the graph $G$ satisfies Hypotheses 1 and 2, the differential operator $(L, \operatorname{Dom}(L))$ is the infinitesimal generator of a unique continuous, conservative, strong Markov process $\left(X_{t}, t \geq 0 ; \mathbb{P}_{x}, x \in \mathfrak{I}\right)$ living in $\mathfrak{I}$.

\subsection{One-dimensional diffusions as diffusions on a graph}

We give now some example of diffusions on simple graphs. Results on onedimension diffusion processes could be found for example in [BRE81]. 
Example 1 (Reflected Brownian motion). The reflected Brownian motion could be seen as a diffusion on a graph with one edge, $\mathbb{R}_{+}$, of infinite length, and one vertex excepted $v^{\infty}$, which is 0 . Its infinitesimal generator is $L f=\mathcal{D}_{0, \mathbb{R}_{+}}^{2} f$ whose domain $\operatorname{Dom}(L)$ is the set of continuous, bounded functions with continuous two first derivatives and such that $f^{\prime}(0+)=0$.

Example 2 (Skew Brownian motion). The skew Brownian motion is the generalization of the Brownian motion, that could be constructed by many ways. On $\mathbb{R}_{+}^{*}$ and $\mathbb{R}_{-}^{*}$, its infinitesimal generator $L$ is given by $L f=\frac{1}{2} f^{\prime \prime}$. Its domains $\operatorname{Dom}(L)$ contains continuous, bounded functions $f$ on $\mathbb{R}$ such that $f \in \mathcal{C}^{2}\left(\mathbb{R}_{+}\right)$and $f \in \mathcal{C}^{2}\left(\mathbb{R}_{-}\right)$for which $\alpha f^{\prime}(0+)=(1-\alpha) f^{\prime}(0-)$ for a given $\alpha \in(0,1)$. We denote the process generated by $(L, \operatorname{Dom}(L))$ by $B^{\alpha}$, and $\alpha$ is called its parameter. If $\alpha=1 / 2$, then $B^{\alpha}$ is a Brownian motion. See WAL78 for this construction.

It could also be described by its speed measure $m(\mathrm{~d} x)=(1-\alpha) \mathrm{d} x$ if $x<0$ and $m(\mathrm{~d} x)=\alpha \mathrm{d} x$ if $x \geq 0$, and its scale function $S(x)=(1-\alpha)^{-1} x$ if $x<0$ and $S(x)=\alpha^{-1} x$ if $x>0$. The process $B^{\alpha}$ is also the solution of the Stochastic Differential Equation (See [HS81]):

$$
\mathrm{d} B_{t}^{\alpha}=\mathrm{d} B_{t}+(2 \alpha-1) \mathrm{d} L_{t}\left(B^{\alpha}\right)
$$

where $B$ is a Brownian motion, and $L_{t}\left(B^{\alpha}\right)$ is the symmetric local time at 0 of the skew Brownian motion.

An alternative description of the skew Brownian motion could be given in term of excursions processes. Let $\left(L_{n}, R_{n}\right)$ be the excursions' intervals of a reflected Brownian motion $B^{\mathrm{r}}$ (these intervals are known to be countable, although they cannot be ordered). We construct a new stochastic process $X$ by the following way. For each excursions, we draw an independent Bernoulli random variable $e_{n} \in\{-1,1\}$, such that $\mathbb{P}\left[e_{n}=1\right]=\alpha$. Then, if $e_{n}=1$, we set $X_{t}=B_{t}^{\mathrm{r}}$ for $t \in\left[L_{n}, R_{n}\right]$. Otherwise, we set $X_{t}=-B_{t}^{\mathrm{r}}$ for $t \in\left[L_{n}, R_{n}\right]$. Thus, $X$ is a skew Brownian motion of parameter $\alpha$. See [IM74] for this construction.

Finally, a last characterization of the SBM could be given in term of excursions' measures: $\mathbb{P}=\alpha \mathbb{P}^{+}+(1-\alpha) \mathbb{P}^{-}$, where $\mathbb{P}, \mathbb{P}^{+}$and $\mathbb{P}^{-}$denote respectively the excursions measures of $B^{\gamma},|B|$ and $-|B|$, where $B$ is a Brownian motion (See [BLU92]).

Example 3 (Process generated by a divergence form operator). Let $a$ be a right-continuous function on $\mathbb{R}$ such that there exist two constants $c$ and $C$ for which $0<c<a(x)<C$ for any $x \in \mathbb{R}$. Besides, $a$ is continuously differentiable on $\mathbb{R}_{+}^{*}$ and $\mathbb{R}_{-}^{*}$ and we assume that $a^{\prime}(0+)$ and $a^{\prime}(0-)$ exists, and that $a^{\prime}$ is bounded. Let also $b$ be a bounded, measurable function on $\mathbb{R}$. 
A. Lejay / Simulating a diffusion on a graph. Application to reservoir engineering

Let $(L, \operatorname{Dom}(L))$ be the infinitesimal generator

$$
L=\frac{1}{2} \frac{\mathrm{d}}{\mathrm{d} x}\left(a(x) \frac{\mathrm{d}}{\mathrm{d} x}\right)+b(x) \frac{\mathrm{d}}{\mathrm{d} x}
$$

whose domain $\operatorname{Dom}(L)$ contains by continuous, bounded functions $f$ on $\mathbb{R}$ such that $f \in \mathcal{C}^{2}\left(\mathbb{R}_{+}\right)$and $f \in \mathcal{C}^{2}\left(\mathbb{R}_{-}\right)$for which

$$
a(0+) f^{\prime}(0+)=a(0-) f^{\prime}(0-) .
$$

It is well known that $(L, \operatorname{Dom}(L))$ is the infinitesimal generator of a continuous, strong Markov process (See for example [LEJ00] for the main properties of such a process), which could then be seen as a diffusion on a graph with two edges, $\mathbb{R}_{+}$and $\mathbb{R}_{-}$.

Note that this process $X$ is solution to the Stochastic Differential Equation

$$
\mathrm{d} X_{t}=\sqrt{a\left(X_{t}\right)} \mathrm{d} B_{t}+\left(\frac{1}{2} a^{\prime}\left(X_{t}\right)+b\left(X_{t}\right)\right) \mathrm{d} t+\frac{a(0+)-a(0-)}{a(0+)+a(0-)} \mathrm{d} L_{t}(X)
$$

where $B$ is a Brownian motion, $L_{t}(X)$ is the symmetric local time at 0 of $X$ (See [LEJ00, Chapter 5]). Thus, $X$ is different from the process $\widehat{X}$ generated by $\widehat{L} L f=\frac{1}{2} a f^{\prime \prime}+\left(\frac{1}{2} a^{\prime}+b\right) f^{\prime}$ by the fact that at 0 , the functions in $\operatorname{Dom}(\widehat{L})$ shall satisfy $f^{\prime}(0+)=f^{\prime}(0-)$. The behavior at 0 is then completely different. The process $X$ tend to go on the side where the diffusion coefficient is maximal, while the process $\widehat{X}$ tend to go on the side where the diffusion coefficient is minimal (See [LEJ01] for a discussion using excursions theory).

Assume now that $b=0$ and that $a$ takes two values $a_{+}$and $a_{-}$respectively in $\mathbb{R}_{+}$and in $\mathbb{R}_{-}^{*}$. Using (44) and the Tanaka formula (See Section 3.6 in [KS91, p. 201] for example), this process is also equal in distribution to $\varphi\left(B^{\gamma}\right)$, where $B^{\gamma}$ is a skew Brownian motion of parameter $\gamma=\sqrt{a_{+}} /\left(\sqrt{a_{+}}+\sqrt{a_{-}}\right)$, and $\varphi(x)$ is equal to $\sqrt{a_{+}} x$ on $\mathbb{R}_{+}$and $\sqrt{a_{-}} x$ on $\mathbb{R}_{-}$(See [OUK90]).

For non constant coefficients, the situation is a bit more complicated, but a description using excursions measures could be given (See [LEJ01]).

\section{4 (Skew) Brownian motion on a graph}

Let $\delta$ be a positive real number satisfying $0<\delta<\inf _{\text {edges } e \text { of } G} \ell_{e}$. At each vertex $v$ and each edge $e$ incident to $v$, we associate the point $x(v, e, \delta)$ on $e$ at distance $\delta$ from $v$, i.e., $d(x(v, e, \delta), v)=\delta$.

We also consider a set of weights $\alpha_{v, e}$ for each vertex $v$ and each edge $e$ incident to $v$. These weights satisfies (2). 
A. Lejay / Simulating a diffusion on a graph. Application to reservoir engineering

Definition 1 (Skew Brownian motion on a graph). A skew Brownian motion on a graph with weights $\left\{\alpha_{v, e}\right\}_{\text {vertex } v, e \sim v}$ is a strong Markov continuous stochastic process $\left(X_{t}, t \geq 0 ; \mathbb{P}_{x}, x \in \mathfrak{I}\right)$ living on $\mathfrak{I}$ and whose evolution is specified by the following description:

- On each $I_{e}, X$ evolves like a one-dimensional Brownian motion until it reaches 0 or $\ell_{e}$, that is the vertices to which $e$ is incident.

- Once on a vertex $v$, for any $\delta \in\left(0, \inf _{\text {edges } e \text { of } G} \ell_{e}\right)$, the distribution of

$$
\tau_{v, \delta}=\inf \left\{t \geq 0 \mid d\left(X_{t}, v\right)=\delta\right\}
$$

is equal to the distribution of the first exit time from $[-\delta, \delta]$ for a Brownian motion starting from 0 . Moreover, $X_{\tau_{v, \delta}}$ is independent from $\tau_{v, \delta}$ and

$$
\mathbb{P}_{v}\left[X_{\tau_{v, \delta}}=x_{v, e, \delta}\right]=\alpha_{v, e}
$$

for any $e \sim v$.

The condition (5) means roughly that once at a vertex, the process $X$ choose the edge it will go at random with a probability $\alpha_{v, e}$. Yet the irregularities of the trajectories of a Brownian motion makes this description mathematically false, since $X$ returns immediately to any vertex it just left. See Example 4 for a correct statement.

Definition 2 (Brownian motion on a graph). A Brownian motion on a graph is a skew Brownian motion on a graph where for any vertex $v$, $\alpha_{v, e}=1 / \operatorname{degree}(v)$, where degree $(v)$ is the number of edges incident to $v$.

A particular example of this process is the generalized skew Brownian motion.

Example 4 (Generalized skew Brownian motion). Let $G$ be a graph composed with $k$ edges, all identified with $\mathbb{R}_{+}$and incident to the vertex corresponding to the point 0 . Thus, this graph could be represented in the complex plane by a set of half-lines emanating from 0 and whose angles with $\mathbb{R}_{+}$are $0 \leq \theta_{1}<\cdots<\theta_{k}<2 \pi$. Let $\alpha_{1}, \ldots, \alpha_{k}$ be some positive real numbers such that $\alpha_{1}+\cdots+\alpha_{k}=1$. Let $B^{\mathrm{r}}$ be a reflected Brownian motion. Denote by $\left(\left[L_{n}, R_{n}\right]\right)_{n \in \mathbb{N}}$ its excursions' intervals. For each interval $\left[L_{n}, R_{n}\right]$, we draw a discrete random variable $e_{n}$ independent from all the other variables such that $\mathbb{P}\left[e_{n}=j\right]=\alpha_{j}$ for $j=1, \ldots, k$. We construct then a new process $X_{t}$ in the complex plane by setting $X_{t}=\left|B_{t}\right| e^{i \theta_{e_{n}}}$ for $t \in\left[L_{n}, R_{n}\right]$. This process $X$ is called a generalized skew Brownian motion. This process was considered first in [WAL78] (See also [BPY]). 
A. Lejay / Simulating a diffusion on a graph. Application to reservoir engineering

In OKA93, T. Okada constructed the density transition function of this process $X$, and shown that its infinitesimal generator is $L f(x)=\frac{1}{2} \mathcal{D}_{0, e^{i \theta_{j}}}^{2} f(x)$ for $x$ in the edge $e^{i \theta_{j}} \mathbb{R}_{+}$, and with the pasting condition: $\sum_{j=1}^{k} \alpha_{j} \mathcal{D}_{0, \mathbb{R}_{+} e^{i \theta_{j}}} f(0)=$ 0 . It is clear that $X$ fits also with our definition of a skew Brownian motion on a graph. The following theorem is then immediately proved using localization arguments.

Theorem 2. Let $G$ be graph satisfying Hypotheses 1 and 2 . For any set of weights $\alpha_{v, e}$, there exists a unique skew Brownian motion on $G$. The infinitesimal generator $(L, \operatorname{Dom}(L))$ of this process is the differential operator $L=\frac{1}{2} \mathcal{D}_{v, e}^{2}$ whose domain $\operatorname{Dom}(L)$ contains bounded functions of class $\mathcal{C}^{2}(\mathfrak{I})$ satisfying (3).

\subsection{Diffusions with constant speed on edges}

We consider now a diffusion $\left(X, \mathbb{P}_{x}\right)$ on a graph $G$ for which the coefficients $a_{e}$ and $b_{e}$ of the infinitesimal generator satisfy

$$
a_{e} \text { is constant on each edge and } b_{e}=0 \text {. }
$$

The study of $X$ could be reduced to the study of a skew Brownian motion on the graph $G$.

Theorem 3. Let $G^{\prime}$ be a copy of $G$. We denote the edge of $G^{\prime}$ corresponding to $e$ by $e^{\prime}$, and $\ell_{e^{\prime}}, a_{e^{\prime}}, \ldots$ correspond to $\ell_{e}, a_{e}, \ldots$ for the graph $G^{\prime}$. We assume that $\ell_{e^{\prime}}=\ell_{e} / \sqrt{a_{e}}$. Let $\Psi$ be the bijection from $\mathfrak{I}$ to $\mathfrak{I}^{\prime}=\cup_{\text {edge } e^{\prime}}\left(e^{\prime}, I_{e^{\prime}}\right)$ defined by $\Psi(e, x)=\left(e^{\prime}, x / \sqrt{a_{e}}\right)$. Then the diffusion $X^{\prime}=\Psi(X)$ is a skew Brownian motion on the graph $G^{\prime}$ with weights $\alpha_{v^{\prime}, e^{\prime}}$ given by

$$
\alpha_{v^{\prime}, e^{\prime}}=\frac{\alpha_{v, e}}{Z_{v^{\prime}} \sqrt{a_{e^{\prime}}}}
$$

where $Z_{v^{\prime}}$ is such that $\sum_{e^{\prime} \sim v^{\prime}} \alpha_{v^{\prime}, e^{\prime}}=1$.

Proof. It is clear that $X^{\prime}$ is a continuous, strong Markov process. Moreover, the infinitesimal generator $\left(L^{\prime}, \operatorname{Dom}\left(L^{\prime}\right)\right)$ of $X^{\prime}$ is defined by $L^{\prime} f=\frac{1}{2} \mathcal{D}_{v_{e}, e}^{2}$, since $X^{\prime}$ behaves like a standard Brownian motion on each interval $I_{e^{\prime}}$.

It remains to study the domain $\operatorname{Dom}\left(L^{\prime}\right)$ of $L^{\prime}$. Remark that a continuous function $f$ belongs to $\operatorname{Dom}\left(L^{\prime}\right)$ if and only if there exists some function $g$ in $\operatorname{Dom}(L)$ such that $g \circ \Phi=f$, where $\Phi$ is the one-to-one function from $\mathfrak{I}^{\prime}$ to $\mathfrak{I}$ defined by $\Phi\left(e^{\prime}, x\right)=\left(e, x \sqrt{a_{e}}\right)$. Using the pasting conditions

$$
\sum_{e \sim v} \alpha_{v, e} \mathcal{D}_{v, e} g(v)=0 \text { and } \sum_{e^{\prime} \sim v^{\prime}} \alpha_{v^{\prime}, e^{\prime}} \mathcal{D}_{v^{\prime}, e^{\prime}} f\left(v^{\prime}\right)=0
$$


A. Lejay / Simulating a diffusion on a graph. Application to reservoir engineering

we are then conducted to

$$
\sum_{e^{\prime} \sim v^{\prime}} \alpha_{v^{\prime}, e^{\prime}} \sqrt{a_{e^{\prime}}} \mathcal{D}_{v^{\prime}, e^{\prime}} g\left(v^{\prime}\right)=0 .
$$

We deduce easily that the weights $\alpha_{v^{\prime}, e^{\prime}}$ shall be given by (17).

The process $X^{\prime}$ is then a skew Brownian motion on the graph $G^{\prime}$ with weights $\alpha_{v^{\prime}, e^{\prime}}$ given by the relation (77).

This relation between $X$ and $X^{\prime}$ could also be found in OKA93], where another approach is used. This theorem generalized the last result given for divergence form operators in Example 3 .

Thus, studying diffusions on graphs with constant speed on each edge may be reduced to the study of skew Brownian motion on graphs using a simple transform.

\section{An exact method to simulate the position of a skew Brownian motion on a graph}

We provide now an exact method to compute the position of a skew Brownian motion on a graph or the first time it hit a set of vertices. By virtue of Theorem 3, this method could also be used for any diffusion with constant speed on each edge.

Remark 1. If we are interested in the first time the particle reaches a set of points, then the method provided by M. Weber in [WEB01a, WEB01b] allows us to speed up the computations by providing the time the particle spend in a "backbone", i.e., a subgraph $\Gamma$ of $G$ linked to $G \backslash \Gamma$ by only one vertex.

The algorithm is quite simple. It follows the construction of the skew Brownian motion in Section 2.4.

We consider the position of the skew Brownian motion $X$ on the graph $G$ at a time $t$. The weights of $X$ are the $\alpha_{v, e}$ 's. This algorithm is easily transformed to compute the first time $X$ hit a set a vertices.

In the following algorithm, all the random variables that are simulated are independent.

Step 1. We assume in a first time that the particle is at the point $x$ in the edge $e$. We identify $x$ with its distance from $v_{e}$.

Let $\tau$ be the first exit time from $\left[0, \ell_{e}\right]$ for a Brownian motion: $\tau=\inf \{t>$ $0 \mid B_{t}=0$ or $\left.B_{t}=\ell_{e}\right\}$. 
A. Lejay / Simulating a diffusion on a graph. Application to reservoir engineering

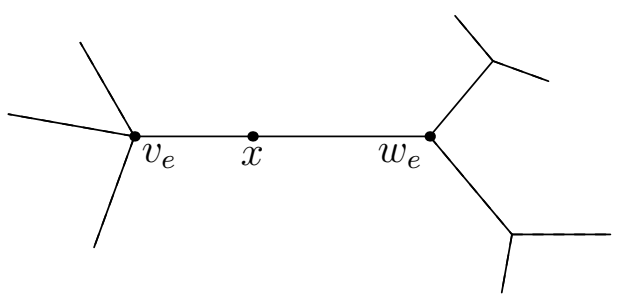

We compute $\gamma=\mathbb{P}_{x}[\tau<t]$, and we go to Step 2 with probability $\gamma$ or to Step 2' with a probability $1-\gamma$.

Step 2. In this case, $\tau<t$ and the Brownian motion $B$ reaches either 0 or $\ell_{e}$ before the time $t$.

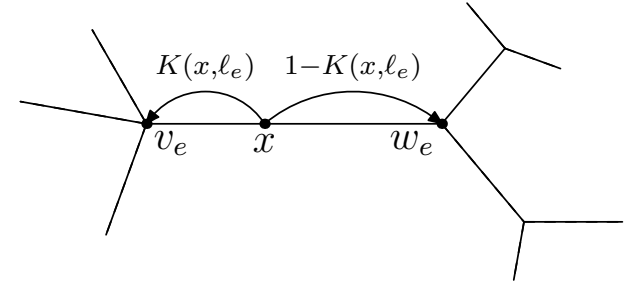

We decide that $B_{\tau}=\ell_{e}$ with a probability

$$
K\left(x, \ell_{e}\right)=\mathbb{P}_{x}\left[B_{\tau}=0 \mid \tau<t\right]=\frac{\mathbb{P}_{x}\left[\tau<t \mid B_{\tau}=0\right]}{\mathbb{P}_{x}[\tau<t]} \times \frac{1-x}{2},
$$

since $\mathbb{P}_{x}\left[B_{\tau}=0\right]=(1-x) / 2$. In this case $B$ exits from the edge $e$ at the vertex $v_{e}$. Otherwise, $B_{\tau}=\ell_{e}$. In this last case, $B$ exits from $e$ at the vertex $w_{e}$.

We also draw a realization $\mathrm{t}$ of a random variable with distribution function $\mathbb{P}_{x}\left[\tau<s \mid \tau<t ; B_{\tau}=\lambda\right]$ for $s \in[0, t]$, where $\lambda$ is either 0 with a probability $K\left(x, \ell_{e}\right)$ or $\ell_{e}$ with a probability $1-K\left(x, \ell_{e}\right)$.

The time final $t$ is set to $t-\mathrm{t}$, and we go to Step 3 .

Step 2'. In this Step, the first exit time $\tau$ from $\left[0, \ell_{e}\right]$ is greater than $t$. Thus, we draw a realization of the position y of the Brownian motion at time $t$ using the following distribution function

$$
F(y)=\mathbb{P}_{x}\left[B_{t}<y \mid t<\tau\right] \text { for } y \in\left[0, \ell_{e}\right]
$$

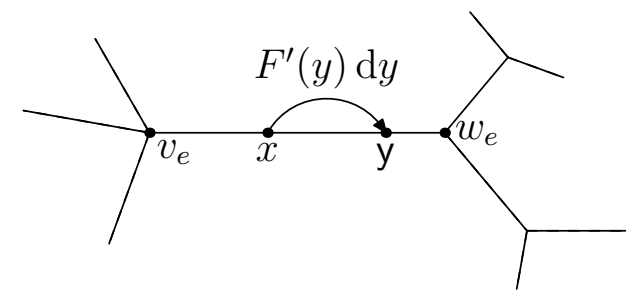

Then, the algorithm stops after having returned the position $(e, \mathrm{y})$. 
Step 3. The Brownian motion is now at a vertex $v$. Let $e_{1}, \ldots, e_{k}$ be an enumeration of all the edges incident to $v$. Fix $\delta \in\left(0, \inf _{i=1, \ldots, k} \ell_{e_{i}}\right)$, and set $x_{i}=x\left(v, e_{i}, \delta\right)$ for $i=1, \ldots, k$, that is, the points at distance $\delta$ from $v$ on each edge $e$ incident to $v$.

Again, set $\gamma=\mathbb{P}_{0}[\tau<t]$, where $\tau$ denotes this time the first exit time from $[-\delta, \delta]$ for a Brownian motion $B: \tau=\inf \left\{t>0 \mid B_{\tau}= \pm \delta\right\}$ when $B_{0}=0$.

We go to Step 4 with a probability $\gamma$ and to Step 4' with a probability $1-\gamma$.

Step 4. In this situation, the diffusion process reaches one of the $x_{i}$ 's before the time $t$. In this case, let $\mathrm{j}$ denotes a realization of a discrete random variable $j$ with distribution $\mathbb{P}[j=i]=\alpha_{i} \stackrel{\text { def }}{=} \alpha_{v, e_{i}}$ for $i=1, \ldots, k$. If $v=v_{e_{\mathrm{j}}}$, we change the value of $(e, x)$ to $\left(e_{\mathrm{j}}, x_{\mathrm{j}}\right)$. Otherwise, we change the value of $(e, x)$ to $\left(e_{\mathrm{j}}, \ell_{e_{\mathrm{j}}}-x_{\mathrm{j}}\right)$.

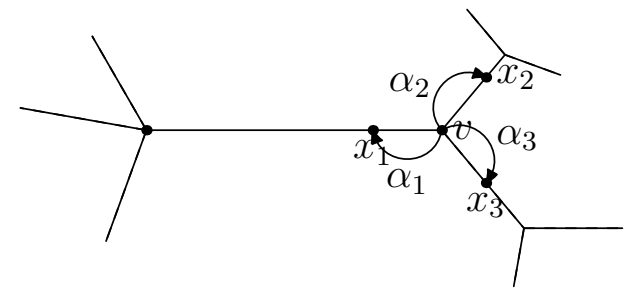

Draw also a realization $t$ of a random variable with distribution function $\mathbb{P}_{0}[\tau<s \mid \tau<t]$ for $s \in[0, t]$. We change the value of $t$ to $t-\mathrm{t}$.

We go to Step 1 with the new values of $t, e$ and $x$.

Step 4'. As in Step 2', the diffusion reaches one of the $x_{i}$ 's after the time $t$. Thus, we compute the position of the diffusion at time $t$ using the following methods.

Let $\mathrm{y}$ be a realization of a random variable with distribution function $F(y)=\mathbb{P}_{0}\left[B_{t}<y \mid t<\tau\right]$ for $y \in[0, \delta]$.

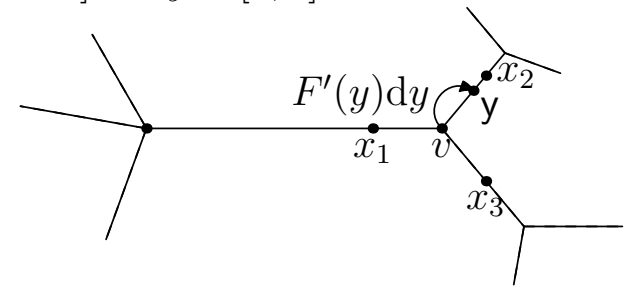

Let also $\mathrm{j}$ be a realization of a discrete random variable $j$ with distribution $\mathbb{P}[j=i]=\alpha_{i}=\alpha_{v, e_{i}}$ for $i=1, \ldots, k$.

If $v=v_{e_{\mathrm{j}}}$, then we set $x$ to y. If $v=w_{e_{\mathrm{j}}}$, then we set $x$ to $\ell_{e_{\mathrm{j}}}-\mathrm{y}$. Then the algorithm stops after having returned $\left(e_{\mathbf{j}}, y\right)$. 
A. Lejay / Simulating a diffusion on a graph. Application to reservoir engineering

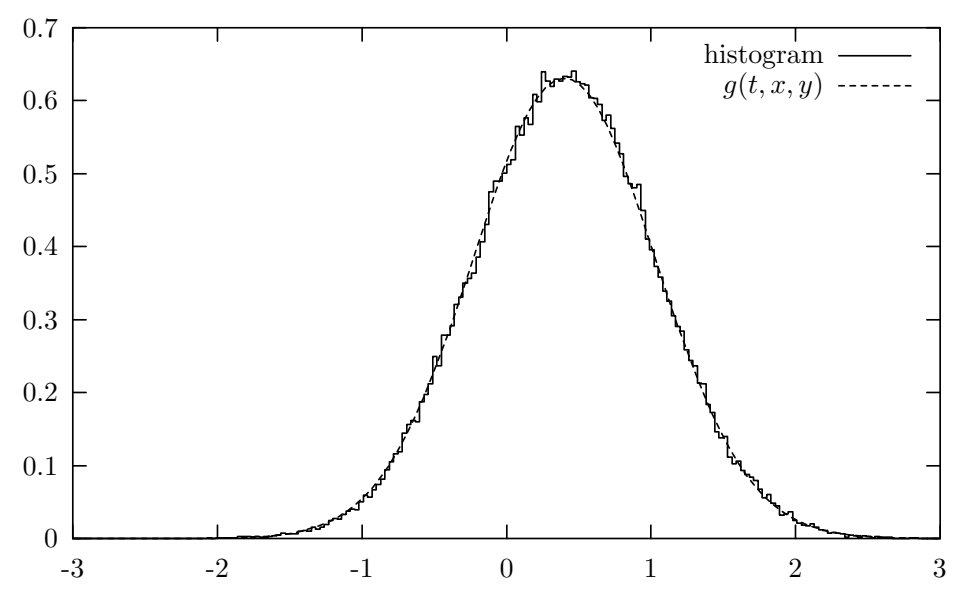

Figure 1: Histogram of the position of the Brownian motion $B$ at time $t=0.4$ with $B_{0}=0.4$, with 100,000 simulations.

\section{Validation}

To test the implementation of our algorithm, we provide two examples for which one knows an explicit density: the position of a Brownian motion at a given time $t$, and the position of the reflected Brownian motion in $[-1,1]$ at time $t$.

\subsection{Position of the Brownian motion}

To deal with our algorithm, we use the integers as vertices. Thus, all the segments have the the same length. In this case, the $\delta$ of Step 3 is equal to 1 . This means that our algorithm performs a (non-homogeneous) random walk on integers, and then use the probability density function of a killed Brownian motion on $[-1,1]$ to compute its position at time $t$.

We draw in Figure 1 a histogram of the position at time $t$ for 100,000 simulations using our algorithm. We see that the histogram matches very well the density $g(t, x, y)=\exp \left(-(x-y)^{2} / 2 t\right) / \sqrt{2 \pi t}$.

\subsection{Position of the reflected Brownian motion}

We also test our algorithm by comparing its result with the density of the reflected Brownian motion on $[-1,1]$, whose density is (See for example [BS96. 
A. Lejay / Simulating a diffusion on a graph. Application to reservoir engineering

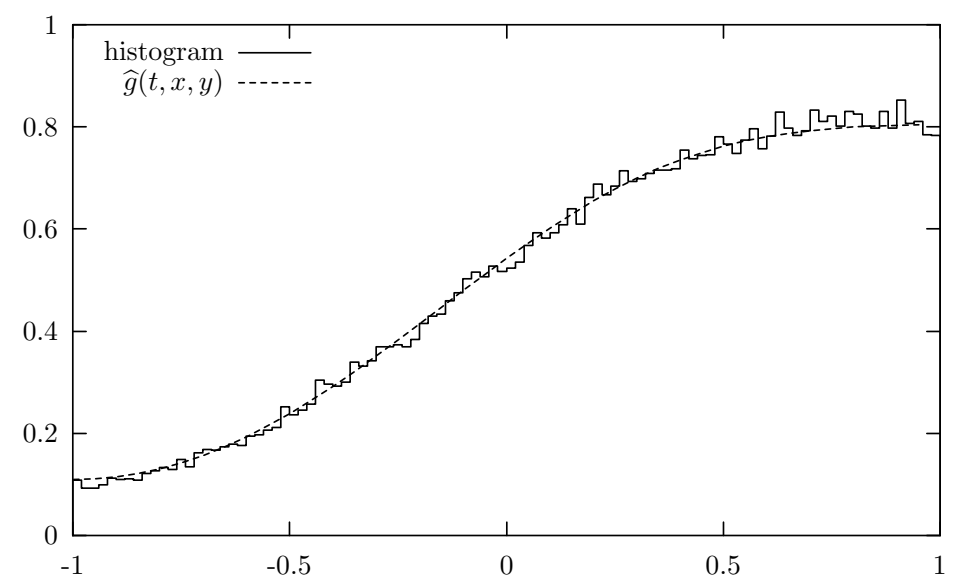

Figure 2: Histogram of the position of the reflected Brownian motion $\widehat{B}$ at time $t=0.4$ with $\widehat{B}_{0}=0.4$, with 100000 simulations.

$\S 7$, p. 105])

$$
\widehat{g}(t, x, y)=\frac{1}{\sqrt{2 \pi t}} \sum_{k=-\infty}^{+\infty}\left(\exp \left(-\frac{(x-y-4)^{2}}{2 t}\right)+\exp \left(-\frac{(x+y-4)^{2}}{2 t}\right)\right) .
$$

We draw in Figure 2 a histogram for 100, 000 simulations to compute the position of the reflected Brownian motion $\widehat{B}$ at time $t=0.4$, when $\widehat{B}_{0}=0.4$. We see that the histogram matches rather well the function $\widehat{g}(t, x, \cdot)$.

For that, we use a graph with 3 vertices, which are $0,-1$ and 1 . As for the simulation of the position of the Brownian motion, we use the fact that $\delta=1$.

\section{Application to geophysics: From fissures to graphs}

\subsection{Motivation}

We are interested in the displacement of a fluid particle in a fissured porous media, due to a diffusion phenomena. The speed at which the particle moves in the fissure is much more higher than the speed in the porous media (also called the matrix). The part of the space occupied by the fissures is denoted by $\Omega_{\mathrm{f}}$, while the part of the space occupied by the matrix is $\Omega_{\mathrm{m}}$. We denote by $S$ the surface between $\Omega_{\mathrm{m}}$ and $\Omega_{\mathrm{f}}$, and we assume that $S$ is piecewise smooth. 
A. Lejay / Simulating a diffusion on a graph. Application to reservoir engineering

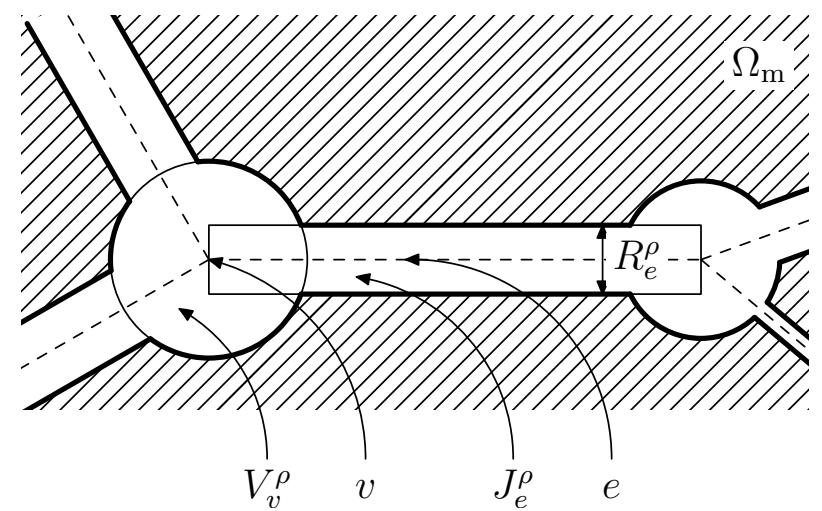

Figure 3: Notations for the fissures' network

The particles of fluids diffuses in $\Omega_{\mathrm{f}}$ at the speed $a_{\mathrm{f}}$. We assume that there is no permeability of the matrix. Thus, the pressure $p(t, x)$ of the fluid is solution to

$$
\begin{aligned}
& \frac{\partial p}{\partial t}(t, x)=\frac{1}{2} a_{\mathrm{f}} \Delta p(t, x) \text { for } t>0, x \in \Omega_{\mathrm{f}}, \\
& \nabla_{n_{\mathrm{f}}} p(t, x)=0 \text { for } t>0, x \in S,
\end{aligned}
$$

where $\nabla_{n_{\mathrm{f}}}$ is the derivative along the normal to $S$ pointing inward $\Omega_{\mathrm{f}}$.

If the initial condition is $p(0, x)=\delta_{y}(x)$, then $p(t, x)$ is the density transition function of a Brownian motion at speed $a_{\mathrm{f}}$ lying in $\Omega_{\mathrm{f}}$ and reflected on $S$.

Thus, $p(t, x)$ may be computed using a Monte Carlo method. Under some assumptions on the domain $\Omega_{\mathrm{f}}$, mainly that the fissures are thin and that the fissures' network is not too dense, we provide a new Monte Carlo method to find the position the particles at time $t$, or to compute when they reach a given set of points.

\subsection{Approximation of the motion of a particle in a fis- sures' network}

We consider now that $G$ could be drawn in $\mathbb{R}^{2}$. This means that $G$ could be seen as a subset of $\mathbb{R}^{2}$ made of the segments of length $\ell_{e}$ which are connected through their endpoints. We keep the identification of an edge $e$, which is now a segment in $\mathbb{R}^{2}$, with a segment $I_{e}=\left[0, \ell_{e}\right] \subset \mathbb{R}$.

Let us introduce our mathematical description of the fissures' network, described also by Figure 3. Given a point $x$ in $\mathbb{R}^{2}$, we could compute its 
orthogonal projection $x^{\perp_{e}}$ on the straight line containing $e$. Given $\rho>0$, let $J_{e}^{\rho}$ be the set

$$
J_{e}^{\rho}=\left\{x \in \mathbb{R}^{2} \mid x^{\perp_{e}} \in I_{e} \text { and }\left\|x-x^{\perp_{e}}\right\|<R_{e}^{\rho}\right\}
$$

where $R_{e}^{\rho}>0$. Thus, $J_{e}^{\rho}$ is called the $R_{e}^{\rho}$-neighbourhood of $e$. We associate to each vertex $v$ a connected subset $V_{v}^{\rho}$ of $\mathbb{R}^{2}$ containing $v$ and such that $J_{e}^{\rho} \cap J_{e^{\prime}}^{\rho} \cap\left(\mathbb{R}^{2} \backslash V^{\rho}\right)=\emptyset$ for any edges $e, e^{\prime}$, where $V^{\rho}=\cup_{\text {vertex }} V_{v}^{\rho}$. We assume that

$$
\Omega_{\mathrm{f}}=\left(\cup_{\text {edge } e} J_{e}^{\rho}\right) \cup\left(\cup_{\text {vertex } v} V_{v}^{\rho}\right) .
$$

With this model, the fissures is made of tubes (the $J_{e}^{\rho}$ 's) of constant width $2 \rho$ which are connected through regions (the $V_{v}^{\rho}$ 's). Moreover, we assume for technical reasons that $\partial V_{v}^{\rho}$ is separated from $\partial V_{v}^{\rho^{\prime}}$ by a positive distance for any $\rho<\rho^{\prime}$.

Hypothesis 3. We assume that $\rho$ is much more smaller than inf edge $_{e} \ell_{e}$, that $\sup _{\text {edge } e} R_{e}^{\rho}$ is of order $\mathrm{O}(\rho)$ and that each $V_{v}^{\rho}$ is included in a ball whose radius is of order $\mathrm{O}(\rho)$.

Now, let $\left(X^{\rho}, \mathbb{P}_{x}\right)$ be the reflected Brownian motion in $\Omega_{\mathrm{f}}$. The condition (8) means that for any point in $\Omega_{\mathrm{f}} \backslash V^{\rho}, x$ belongs to only one $J_{e}^{\rho}$. Let $\varphi^{\rho}$ be a continuous function from $\Omega_{\mathrm{f}}$ to $\mathbb{R}^{2}$ such that $\varphi^{\rho}(x)=x^{\perp_{e}}$ if $x$ belongs to $J_{e}^{\rho} \backslash V^{2 \rho}$ and that $\varphi^{\rho}\left(V_{v}^{\rho}\right) \subset B(v, \rho)$, where $B(v, \rho)$ is a ball of center $v$ and radius $\rho$.

The stochastic process $\varphi^{\rho}\left(X^{\rho}\right)$ is not necessarily a Markov process. However, it converges in distribution to some simple process.

Theorem 4 ([FW93]). As $\rho \rightarrow 0$, the process $\varphi^{\rho}\left(X^{\rho}\right)$ converges in distribution in the space of continuous functions to the skew Brownian motion on the graph $G$ with weights

$$
\alpha_{v, e}=\lim _{\rho \rightarrow 0} \frac{R_{e}^{\rho}}{\sum_{e^{\prime} \sim v} R_{e^{\prime}}^{\rho}} .
$$

This theorem means that the time spend by the process $X^{\rho}$ in the intersections $V_{v}^{\rho}$ around each vertex is quite small and may be neglected.

For practical use, the position of the process $X^{\rho}$ may be found using a Monte Carlo method to simulate a diffusion on a graph, such as the one given in Section 3 . 
A. Lejay / Simulating a diffusion on a graph. Application to reservoir engineering

\section{A Formulae for simulating random variables}

We provide in this appendix the formulae that are needed to simulate random variables in our algorithm.

A random variable with an invertible distribution function $F$ which is explicitly known is easily simulated by the following way: Let $\mathrm{u}$ be a realization of a uniform random variable on $[0,1]$. Then $F^{-1}(\mathrm{u})$ is a realization of such a random variable.

\section{A.1 The exit time from an interval}

Let $\left(B_{t}, t \geq 0 ; \mathbb{P}_{x}, x \in \mathbb{R}\right)$ be a standard one-dimensional Brownian motion, and let us denote by $\tau$ its first exit time from $[-1,1]$. Obviously, with the scaling property of the Brownian motion, it is sufficient to simulate $\tau$ in order to obtain simulation of the exit time from any interval.

We set $G(t)=\mathbb{P}_{x}[\tau<t]$. Then the Laplace transform of $\tau$ is

$$
\mathbb{E}_{x}\left[e^{-\lambda \tau}\right]=\frac{\operatorname{ch}((1-x) \sqrt{2 \lambda})}{\operatorname{ch}(\sqrt{2 \lambda})}
$$

Inverting this, we have the following density distribution function (See for example Formula 1-3.0.2 in [BS96, p. 172])

$$
G^{\prime}(t)=\sum_{k=-\infty}^{+\infty}\left(\frac{x+1+4 k}{\sqrt{2 \pi} t^{3 / 2}} e^{-(x+1+4 k)^{2} / 2 t}+\frac{1-x+4 k}{\sqrt{2 \pi} t^{3 / 2}} e^{-(1-x+4 k)^{2} / 2 t}\right) .
$$

Thus, the random variable with distribution function $\mathbb{P}_{x}[\tau<s \mid \tau<t]$ is easily simulated using the distribution function $G(s) / G(t)$ for $s \in[0, t]$.

We are also required to simulate a random variable with distribution function $\mathbb{P}_{x}\left[\tau<s \mid \tau<t ; B_{\tau}=\lambda\right]$ where $\lambda=-1$ or $\lambda=1$. Let us remark that by symmetry of the Brownian motion,

$$
\mathbb{P}_{x}\left[\tau<s \mid \tau<t ; B_{\tau}=-1\right]=\mathbb{P}_{-x}\left[\tau<s \mid \tau<t ; B_{\tau}=1\right]
$$

and that $\mathbb{P}_{x}\left[\tau<s \mid \tau<t ; B_{\tau}=1\right]=H(s) / H(t)$ for $s \in[0, t]$, with

$$
H(t)=\mathbb{P}_{x}\left[\tau<t \mid B_{\tau}=1\right] .
$$

The derivative $H^{\prime}$ of $H$ is (See formula 1-3.0.6(b) in [BS96, p. 172] for example)

$$
H^{\prime}(t)=\sum_{k=-\infty}^{+\infty} \frac{1-x+4 k}{\sqrt{2 \pi} t^{3 / 2}} e^{-(1-x+4 k)^{2} / 2 t}
$$


A. Lejay / Simulating a diffusion on a graph. Application to reservoir engineering

\section{A.2 The position at a given time before the first exit time}

We also need to simulate the position of a Brownian motion at time $t$ given it has not exit from an interval at this time. Again using the scaling property of the Brownian motion, we could consider that this interval is $[-1,1]$.

Let $\widehat{F}$ be the function

$$
\widehat{F}(y)=\mathbb{P}_{x}\left[B_{t}<y \mid t<\tau\right]=\frac{F(y)}{G(t)} \text { for } y \in[-1,1]
$$

with $F(y)=\mathbb{P}_{x}\left[B_{t}<y ; t<\tau\right]$.

The derivative $F^{\prime}(y)$ of $F$ is equal to the transition density $p(t, x, y)$ of the killed Brownian motion. Thus,

$$
\begin{aligned}
F^{\prime}(y)=\frac{1}{\sqrt{2 \pi t}} \sum_{k=-\infty}^{+\infty}\left(\exp \left(-\frac{(x-y-4 k)^{2}}{2 t}\right)\right. & \left.-\exp \left(-\frac{(x+y+2+4 k)^{2}}{2 t}\right)\right) .
\end{aligned}
$$

Besides, using the spectral representation of $p(t, x, y)$,

$$
F^{\prime}(y)=\frac{1}{2} \sum_{k=1}^{+\infty} \exp \left(-\frac{k^{2} \pi^{2}}{8} t\right) \sin \left(\frac{k \pi}{2}(x+1)\right) \sin \left(\frac{k \pi}{2}(y+1)\right) .
$$

One can integrate (9) and (10) to obtain two expressions of $F$ as series. Thus, $F$ is easily inverted using the Newton-Raphson method. We remark that from a numerical point of view, it is worth using (9) when the time is small and (10) when the time is large. Finally, let us remark that every series that are involved in the computation of $F$ and $F^{\prime}$ converge very quickly.

\section{References}

[BPY] M. Barlow, J. Pitman and M. Yor. On Walsh's Brownian motions. in Séminaire de Probabilités, XXIII, vol. 1372 of Lecture Notes in Math., pp. 275-293. Springer, Berlin, 1989.

[BLU92] R.M. Blumenthal. Excursions of Markov Processes. Probability and Its Applications. Birkhäuser, 1992. 5

[BRE81] L. Breiman. Probability. Addison-Wesley, 1981. 4 
A. Lejay / Simulating a diffusion on a graph. Application to reservoir engineering

[BS96] A.N. Borodin and P. Salminen. Handbook of Brownian Motion - Facts and Formulae. Probability and Its Applications. Birkhäuser, 1996. 13, 16

[CL01] F. Campillo and A. Lejay. A Monte Carlo Method to Compute the exchange coefficient in the double porosity model. Monte Carlo Methods Appl., 7:1-2, pp. 65-72, 2001. Conference proceedings of Monte Carlo 2000. 2

[CL02] F. Campillo and A. Lejay. A Monte Carlo method without grid for a fractured porous domain model. Monte Carlo Methods Appl., 8:2, pp. 129-148, 2002. 2

[DJ93] D. S. DEAn and K. M. Jansons. Brownian excursions on combs. J. Statist. Phys., 70:5-6, pp. 1313-1332, 1993. 2

[FS00] M. Freidlin and S.-J. Sheu. Diffusion processes on graphs: stochastic differential equations, large deviation principle. Probab. Theory Related Fields, 116:2, pp. 181-220, 2000. 2

[FW93] M.I. Freidlin and A.D. Wentzell. Diffusion processes on graphs and the averaging principle. Ann. Probab., 21:4, pp. 22152245, 1993. 2, 4, 15

[FW98] M. Freidlin and M. WEBER. Random perturbations of nonlinear oscillators. Ann. Probab., 26:3, pp. 925-967, 1998. 2

[HS81] J.M. HARrison and L.A. Shepp. On Skew Brownian Motion. Ann. Probab., 9:2, pp. 309-313, 1981. 5

[IM74] K. Itô and H.P. McKean. Diffusion and Their Sample Paths. Springer-Verlag, $2^{\text {nd }}$ edition, 1974. 5

[KS91] I. Karatzas and S.E. Shreve. Brownian Motion and Stochastic Calculus. Springer-Verlag, $2^{\text {nd }}$ edition, 1991. 6

[LEJ00] A. LEJAY. Méthodes probabilistes pour l'homogénéisation des opérateurs sous forme-divergence : cas linéaires et semi-linéaires. PhD thesis, Université de Provence, Marseille, France, 2000.

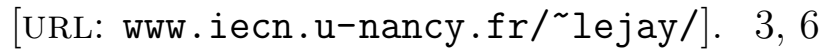

[LEJ01] A. LEJAY. On the decomposition of excursions measures of processes whose generators have diffusion coefficients discontinuous 
A. Lejay / Simulating a diffusion on a graph. Application to reservoir engineering

at one point. Markov Process. Related Fields, 8:1, pp. 117-126, 2001. 3, 6

[LUM84] G. LumER. Équations de diffusion générales sur des réseaux infinis. In Seminar on potential theory, Paris, No. 7, vol. 1061 of Lecture Notes in Math., pp. 230-243. Springer, Berlin, 1984. 2

[OKA93] T. OKADA. Asymptotic behavior of skew conditional heat kernels on graph networks. Canad. J. Math., 45:4, pp. 863-878, 1993. 8, 9

[OUK90] Y. OUKnINE. "Skew-Brownian motion" and derived processes. Theory of Probab. App., 35:1, pp. 163-169, 1990. 6

[WAL78] J.B. WALSH. A diffusion with a discontinuous local time. In Temps locaux, Astérisques, pp. 37-45. Société Mathématique de France, 1978. 5, 7

[WEB01a] M. WEBER. Correction note: "On occupation time functionals for diffusion processes and birth-and-death processes on graphs". Ann. Appl. Probab., 11:3, pp. 1003, 2001. 9

[WEB01b] M. WEBER. On occupation time functionals for diffusion processes and birth-and-death processes on graphs. Ann. Appl. Probab., 11:2, pp. 544-567, 2001. 9] 